\title{
一种利用精确纳米组装结构调控水下产气速率的普适方法
}

俞书宏

中国科学技术大学化学系, 合肥 230026

\section{A General Strategy for Accelerating Fuel Gas Evolution on Nanoparticle Assemblies}

\section{Shuhong Yu}

Department of Chemistry, University of Science and Technology of China, Hefei 230026, P. R. China.

Emails: shyu@ustc.edu.cn.

Published online: January 13, 2020.
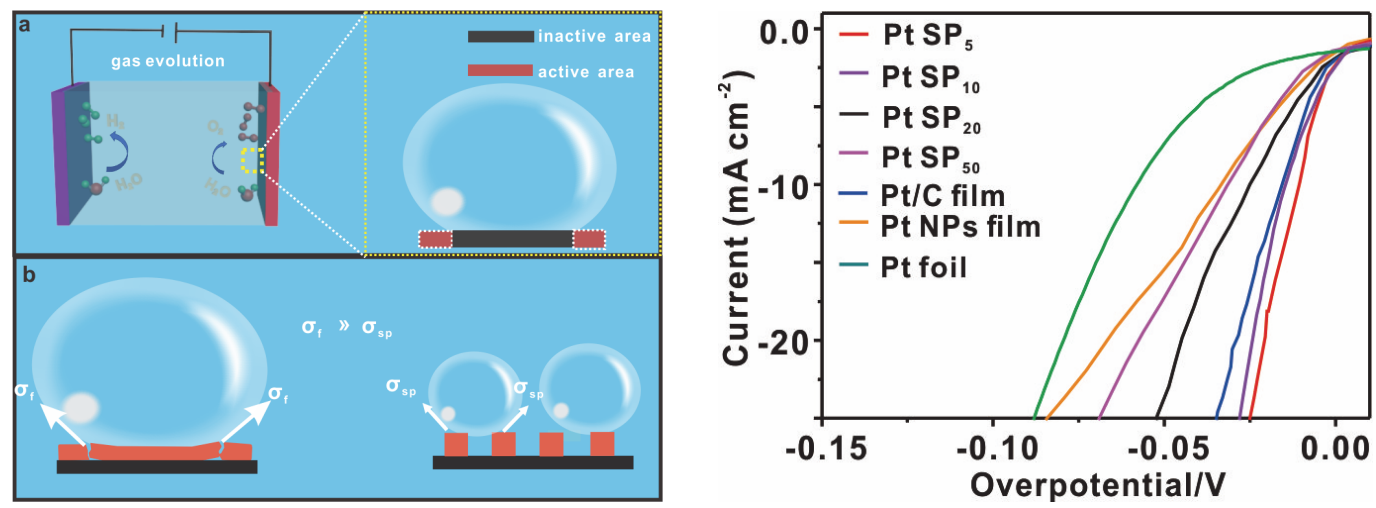

条带结构用于水下产气和条带电极电催化产氢的优势。

由于能源气体如氢气 $\left(\mathrm{H}_{2}\right)^{1}$, 一氧化碳 $(\mathrm{CO})^{2}$ 和氧气 $\left(\mathrm{O}_{2}\right)^{3}$ 在可持续和高效能源系统中的潜在应 用, 目前正引起全世界的关注。目前大多数工作都 致力于设计和研发高效的催化剂以达水下高效电 催化产气的效果, 例如设计特殊晶体构型的金属 及金属氧化物 ${ }^{4}$, 碳材料负载少量的贵金属等等 ${ }^{5}$ 。 大多数制备的催化剂是以滴涂的方式在电极表面 生成薄膜, 在优化后的电压条件下进行电解。除了 考虑异相催化剂的反应活性, 在水下生成的气体 产物会黏附在催化剂膜的表面 ${ }^{6}$, 影响反应物与催 化剂的传质过程, 同时生成的大尺寸气泡会继续 黏附在催化剂表面 ${ }^{7}$, 生成许多非活性位点, 减小 了催化剂的反应面积。催化剂的稳定性是工业生 产顺利进行的条件。黏附生长的气泡直至浮力大 于黏附力时才能脱离催化剂表面, 此时会对催化 剂膜产生较大的拉力, 经长时间电解后, 催化剂由 于气泡的持续作用力会从电极表面脱落, 最终会
影响催化效果。因此从该变催化剂膜的角度出发, 设计催化体系既能加快反应速率又能保持催化剂 长久物理稳定性是解决问题的关键。

近日, 中国科学院化学研究所王铁研究员等 人在前期模板辅助的打印策略组装工作的基础上 (Advanced Materials) ${ }^{8}$, 将胶束型的纳米颗粒成功 组装成条带状的微结构, 与传统的滴涂薄膜相比 实现了更快的产气速率和更高的物理稳定性, 相 关工作发表在Journal of the American Chemical Society ${ }^{9}$ 。作者通过小角X射线衍射证实了微结 构呈现长程有序的超晶格结构。通过改变模板的 参数, 可以调控条带间的间隙宽度。

作者首先将铂 $(\mathrm{Pt})$ 纳米颗粒组装成条带结构 (Pt SP) 用于电催化产氢, 证实了窄间隙的 $\mathrm{Pt} \mathrm{SP}_{5}$ 在 酸性条件下的产氢速率是直接滴涂的Pt NPs薄膜 的5.16倍。作者利用高速摄像机探究了反应过程中 气泡生长行为, 发现气泡在窄间隙的 $\mathrm{Pt} \mathrm{SP}_{5}$ 上是以 
类似 “Cassie” 状态生长并且快速脱离, 而当条带 间隙增大后，气泡以 “Wenzel” 状态陷入间隙中， 并且越长越大直至浮力大于黏附力时才脱离催化 剂表面。结合动态拉伸仪和理论分析, 证实气泡在 $\mathrm{Pt} \mathrm{SP}_{5}$ 表面的黏附力仅有 $2 \mu \mathrm{N}$, 且 $\mathrm{Pt} \mathrm{SP}_{5}$ 具有更好 的应力分散效果 ${ }^{10}$, 因此与滴涂的Pt NPs 薄膜相 比, Pt $\mathrm{SP}_{5}$ 这种条带电极展现了更好的物理稳定 性。此外, 模板辅助打印技术的优势使组成纳米颗 粒或分子的组成、大小和形状具有很大的灵活性， 因此具有通用性, 有望用于析氧反应(OER)和二氧 化碳电化学还原生产 $\mathrm{CO}$ 。

\section{References}

(1) Gao, Q.; Wu, R.; Liu, Y.; Zheng, Y. R.; Li, Y.; Shang, L. M.; Ju, Y. M.; Gu, C.; Zheng, X. S.; Liu, J. W.; et al. Research 2019, 11, 2019. doi: $10.34133 / 2019 / 8078549$

(2) Zhuang, T. T.; Liang, Z. Q.; Seifitokaldani, A.; Li, Y.; De Luna, P.; Burdyny, T.; Che, F.; Meng, F.; Min, Y.; Quintero-Bermudez, R.; et al. Nat. Catal. 2018, 1 (6), 421. doi: 10.1038/s41929-018-0084-7
(3) Duan, Y.; Yu, Z. Y.; Hu, S. J.; Zheng, X. S.; Zhang, C. T.; Ding, H. H.; Hu, B. C.; Fu, Q. Q.; Yu, Z. L.; Zheng, X.; et al. Angew. Chem. Int. Ed. 2019, 58 (44), 15772. doi: 10.1002/anie.201909939

(4) Zeng, M.; Li, Y. G. J. Mater. Chem. A. 2015, 3, 14942. doi: 10.1039/C5TA02974K

(5) Song, Q.; Qiao, X.; Liu, L.; Xue, Z.; Huang, C.; Wang, T. Chem. Commun. 2019, 55 (7), 965. doi: 10.1039/C8CC09624

(6) Sides. P. J.; Tobias, C. W. J. Electrochem. Soc. 1985, 132, 583. doi: $10.1149 / 1.2113910$

(7) Lu, Z. Y.; Zhu, W.; Yu, X. Y.; Zhang, H. C.; Li, Y. J.; Sun, X. M.; Wang, X. W.; Wang, H.; Wang, J. M.; Luo, J.; et al. Adv. Mater. 2014, 26, 2683. doi: 10.1002/adma.201304759

(8) Feng, J.; Song, Q.; Zhang, B.; Wu, Y.; Wang, T.; Jiang, L. Adv. Mater. 2017, 29 (46), 1703143. doi: 10.1002/adma.201703143

(9) Song, Q.; Xue, Z.; Liu, C.; Qiao, X.; Liu, L.; Huang, C.; Liu, K.; Li, X.; Lu, Z.; Wang, T. J. Am. Chem. Soc. 2019, doi: $10.1021 /$ jacs. 9 b10388

(10) Buehler, M. J.; Yao, H. M.; Gao, H. J.; Ji, B. H. Model. Simu.l Mater. Sci. Eng. 2006, 14, 799. doi: 10.1088/0965-0393/14/5/001 